\title{
Connection situations under uncertainty
}

\author{
Stefano Moretti ${ }^{1}$, S. Zeynep Alparslan Gök ${ }^{2,5}$, Rodica Branzei ${ }^{3}$ \\ and Stef Tijs ${ }^{4}$
}

Abstract: This paper deals with cost allocation problems arising from connection situations where edge costs are closed intervals of real numbers. To solve such problems, we extend classical solutions from the theory of minimum cost spanning tree games. We study the properties of such solutions and suggest how to use them under uncertainty conditions.

Key-words: cooperative cost games, minimum cost spanning tree situations, robustness, worst-case scenario, input interval data, uncertainty.

\section{Introduction}

A connection situation takes place in the presence of a group of agents, each of which needs to be connected directly or via other agents to a source, where connections are costly and the configuration of links which minimizes the total cost of connection is provided by a minimum cost spanning tree (mcst). However, finding an mest does not guarantee that it is going to be really implemented: agents must still support the cost of the mcst and then

\footnotetext{
${ }^{1}$ Unit of Molecular Epidemiology, National Cancer Research Institute of Genoa, Italy, e-mail: stefano.moretti@istge.it

${ }^{2}$ Institute of Applied Mathematics, Middle East Technical University, Ankara, Turkey and Süleyman Demirel University, Faculty of Arts and Sciences, Department of Mathematics, Isparta, Turkey, e-mail: alzeynep@metu.edu.tr

${ }^{3}$ Faculty of Computer Science, "Alexandru Ioan Cuza" University, Iaşi, Romania, email: branzeir@infoiasi.ro

${ }^{4}$ CentER and Department of Econometrics and OR, Tilburg University, Tilburg, The Netherlands and Department of Mathematics, University of Genoa, Italy, e-mail: S.H.Tijs@uvt.nl

${ }^{5}$ This author acknowledges the support of TUBITAK (Turkish Scientific and Technical Research Council) and hospitality of Department of Mathematics, University of Genoa, Italy.
} 
a cost allocation problem must be addressed. This cost allocation problem was introduced by Claus and Kleitman in 1973 and has been studied with the aid of cooperative game theory since the basic paper of Bird (1976).

Since that basic paper, many authors have noted that this kind of cost allocation problems may arise from many different physical networks, as telephone lines, highways, electric power systems, computer chips, water delivery systems, rail lines etc. On the other hand, it seems to us that, in many real applications, to retrieve the information about the exact cost of all of the links of a network is often an impossible mission. We argue that it is more likely to imagine real connection situations where the costs of links are traceable at a level of uncertainty, i.e. only the range of the costs is known, and no probability information on the realization of costs is given.

The problem to find an optimal spanning tree on a weighted graph where edge costs are interval numbers has been widely studied in literature. We mainly follow the approaches introduced in Yaman et al. (1999; 2001) to solve the optimization problem. See also Montemanni (2006).

As in the classical case where edge costs are real numbers, also in the situation where edge costs are intervals of real numbers a cost allocation problem arises. With the goal to study this kind of cost allocation problems, in this paper we extend the notion of conservative Construct and Charge rules, which have been introduced in Moretti et al. (2008), and we study their properties under uncertainty conditions.

To the best of our knowledge, this is the first attempt to study a cost allocation problem arising from connection situation under uncertainty. Suijs (2003) studied mcst problems in which the connection costs are represented by random variables. In our paper, costs are not random variables, but just closed intervals of real numbers with no probability distribution attached.

In Section 2 some preliminaries and basic notations are introduced. In Section 3 the notion of minimum interval cost spanning tree situation is presented and the concept of mest situation associated to different scenarios is given. Section 4 deals with minimum interval cost spanning tree situations 
where a permanent tree exists, i.e. a spanning tree which is of minimum cost under all possible scenarios. In Section 5 a pessimistic approach to solve the optimization problem and the consequent pessimistic cost game are studied. In a similar way, Section 6 deals with an optimistic approach.

Permanent trees and trees selected by the pessimistic and optimistic approach are ex-ante (before the construction of the optimal tree) choices. Differently, Section 7 introduce ex-post procedures to adjust the cost allocations provided by ex-ante choices. Section 8 concludes with some open problems.

\section{Preliminaries}

In this section we recall some basic definitions and results regarding minimum cost spanning tree (mcst) situations.

An (undirected) graph is a pair $\langle V, E\rangle$, where $V$ is a set of vertices or nodes and $E$ is a set of edges $e$ of the form $\{i, j\}$ with $i, j \in V, i \neq j$. The complete graph on a set $V$ of vertices is the graph $\left\langle V, E_{V}\right\rangle$, where $E_{V}=\{\{i, j\} \mid i, j \in V$ and $i \neq j\}$.

A mcst situation is a situation where a set $N=\{1, \ldots, n\}$ of agents is willing to be connected as cheap as possible to a source (i.e. a supplier of a service) denoted by 0 , based on a given weight (or cost) system of connection. In the sequel we use also the notation $N^{\prime}=N \cup\{0\}$, and $w$ for the weight function, i.e. a map which assigns to each edge $e \in E_{N^{\prime}}$ a nonnegative number $w(e)$ representing the weight or cost of edge $e$. We denote an mcst situation with set of users $N$, source 0 , and weight function $w$ by $<N^{\prime}, w>$ (or simply $w)$. Further, we denote by $\mathcal{W}^{N^{\prime}}$ the set of all of the mest situations $<N^{\prime}, w>$ (or $w$ ) with node set $N^{\prime}$. A path between $i$ and $j$ in a graph $\left\langle N^{\prime}, E\right\rangle$ is a sequence of nodes $i=i_{0}, i_{1}, \ldots, i_{k}=j, k \geq 1$, such that all the edges $\left\{i_{s}, i_{s+1}\right\} \in E$, for each $s \in\{0, \ldots, k-1\}$, are distinct edges. A cycle in $<N^{\prime}, E>$ is a path from $i$ to $i$ for some $i \in N^{\prime}$. Two nodes $i, j \in N^{\prime}$ are connected in $\left\langle N^{\prime}, E\right\rangle$ if $i=j$ or if there exists a path between $i$ and $j$ in $E$. A connected component of $N^{\prime}$ in a graph $\left\langle N^{\prime}, E\right\rangle$ is a maximal subset 
of $N^{\prime}$ with the property that any two nodes in this subset are connected in $<N^{\prime}, E>$.

The cost of a network $\Gamma \subseteq E_{N^{\prime}}$ is $w(\Gamma)=\sum_{e \in \Gamma} w(e)$. A network $\Gamma$ is a spanning network on $S^{\prime}=S \cup\{0\}$, with $S \subseteq N$, if for every $e \in \Gamma$ we have $e \in E_{S^{\prime}}$ and for every $i \in S$ there is a path in $\left\langle S^{\prime}, \Gamma>\right.$ from $i$ to the source. For any mcst situation $w \in \mathcal{W}^{N^{\prime}}$ it is possible to determine at least one spanning tree on $N^{\prime}$, i.e. a spanning network without cycles on $N^{\prime}$, of minimum cost; each spanning tree of minimum cost is called an mcst for $N^{\prime}$ in $w$ or, shorter, an mest for $w$. In the following we will denote by $\mathcal{S T}^{N^{\prime}}$ the set of all spanning tree on $N^{\prime}$.

The set $\Sigma_{E_{N^{\prime}}}$ of linear orders on $E_{N^{\prime}}$ is defined as the set of all bijections $\sigma:\left\{1, \ldots,\left|E_{N^{\prime}}\right|\right\} \rightarrow E_{N^{\prime}}$, where $\left|E_{N^{\prime}}\right|$ is the cardinality of the set $E_{N^{\prime}}$. For each mcst situation $\left\langle N^{\prime}, w>\right.$ there exists at least one linear order $\sigma \in \Sigma_{E_{N^{\prime}}}$ such that $w(\sigma(1)) \leq w(\sigma(2)) \leq \ldots \leq w\left(\sigma\left(\left|E_{N^{\prime}}\right|\right)\right)$. For any $\sigma \in \Sigma_{E_{N^{\prime}}}$ we define the set

$$
K^{\sigma}=\left\{w \in \mathbb{R}_{+}^{E_{N^{\prime}}} \mid w(\sigma(1)) \leq w(\sigma(2)) \leq \ldots \leq w\left(\sigma\left(\left|E_{N^{\prime}}\right|\right)\right)\right\}
$$

The set $K^{\sigma}$ is a cone in $\mathbb{R}_{+}^{E_{N^{\prime}}}$, which we call the Kruskal cone with respect to $\sigma$.

Let $N=\{1, \ldots, n\}$ and $\Delta(N)=\left\{x \in \mathbb{R}_{+}^{N} \mid \sum_{i \in N} x_{i}=1\right\}$. The set of $n$-vectors of edges which form a spanning tree on $N^{\prime}$ is denoted by $\mathcal{E}_{N^{\prime}}$, i.e. $\quad \mathcal{E}_{N^{\prime}}=\left\{\left(a_{1}, \ldots, a_{n}\right) \in\left(E_{N^{\prime}}\right)^{n} \mid\left\{a_{1}, \ldots, a_{n}\right\} \in \mathcal{S} \mathcal{T}^{N^{\prime}}\right\}$. Note that the number of edges which form a spanning tree on $N^{\prime}$ is $n$.

Given an element $\mathbf{a}=\left(a_{1}, \ldots, a_{n}\right) \in\left(E_{N^{\prime}}\right)^{n}$, we denote by $\mathbf{a}_{\mid j}$ the restriction of $\mathbf{a}$ to the first $j$ components, that is $\mathbf{a}_{\mid j}=\left(a_{1}, \ldots, a_{j}\right)$ for each $j \in N$. Further, for each $j \in N$, we denote by $\Pi\left(\mathbf{a}_{\mid j}\right)$ the partition of $N^{\prime}$ such that

$$
\Pi\left(\mathbf{a}_{\mid j}\right)=\left\{T \subseteq N^{\prime} \mid T \text { is a connected component in }<N^{\prime},\left\{a_{1}, \ldots, a_{j}\right\}>\right\} .
$$

Let $\theta \in \Theta\left(N^{\prime}\right)$, where $\Theta\left(N^{\prime}\right)$ is the family of partitions of $N^{\prime}$, and let $T \subseteq N^{\prime}$. If $T$ is a subset of a certain element of the partition $\theta$, we denote this element as $S(\theta, T)$. A charge system $\mathcal{C}$ on $N$ is a set of functions $\mathcal{C}=$ 
$\left\{C^{1}, \ldots, C^{n}\right\}$ with $C^{j}:\left\{\mathbf{a}_{\mid j} \mid \mathbf{a} \in \mathcal{E}_{N^{\prime}}\right\} \rightarrow \Delta(N)$ for each $j \in N$ satisfying the following properties:

(Connection property): $\quad C_{i}^{j}\left(\mathbf{a}_{\mid j}\right)=0$ for each $i \in S\left(\Pi\left(\mathbf{a}_{\mid j-1}\right),\{0\}\right)$, each $j \in N$, and each $\mathbf{a}=\left(a_{1}, \ldots, a_{n}\right) \in \mathcal{E}_{N^{\prime}}$;

(Involvement property): $\quad C_{i}^{j}\left(\mathbf{a}_{\mid j}\right)=0$ for each $i \in N \backslash S\left(\Pi\left(\mathbf{a}_{\mid j}\right), a_{j}\right)$ each $j \in N$, and each $\mathbf{a}=\left(a_{1}, \ldots, a_{n}\right) \in \mathcal{E}_{N^{\prime}}$;

(Total aggregation property): $\sum_{j=1}^{n} C_{i}^{j}\left(\mathbf{a}_{\mid j}\right)=1$ for each $i \in N$, and each $\mathbf{a}=\left(a_{1}, \ldots, a_{n}\right) \in \mathcal{E}_{N^{\prime}}$.

Based on the notion of charge systems, we may define special allocation rules for spanning trees where costs are shared among agents during the edge by edge construction of the tree. Let $\mathcal{C}=\left\{C^{1}, \ldots, C^{n}\right\}$ be a charge system on $N$ and Let $\sigma \in \Sigma_{E_{N^{\prime}}}$. We define the set $\mathcal{E}_{N^{\prime}}^{\sigma}=\left\{\mathbf{a} \in \mathcal{E}_{N^{\prime}}: \sigma^{-1}\left(a_{1}\right) \leq\right.$ $\left.\sigma^{-1}\left(a_{2}\right) \leq \ldots \leq \sigma^{-1}\left(a_{n}\right)\right\}$. The tree dependent allocation protocol $\kappa^{\mathcal{C}, \sigma}$ based on $\mathcal{C}$ and $\sigma$ is the map $\chi^{\mathcal{C}, \sigma}: K^{\sigma} \times \mathcal{E}_{N^{\prime}}^{\sigma} \rightarrow \mathbb{R}^{N}$ given by

$$
\kappa^{\mathcal{C}, \sigma}(w, \mathbf{a})=\sum_{r=1}^{n} w(\sigma(r)) C^{r}\left(\mathbf{a}_{\mid j}\right)
$$

for each mcst situation $w$ in the Kruskal cone $K^{\sigma}$ and each $\mathbf{a} \in \mathcal{E}_{N^{\prime}}^{\sigma}$.

According to Moretti et al. (2008), the aggregate contribution of the charge system $\mathcal{C}$ on $\mathbf{a}_{\mid j}$, for each $j \in N$ and for each $\mathbf{a}=\left(a_{1}, \ldots, a_{n}\right) \in \mathcal{E}_{N^{\prime}}$, is the $n$-vector $\mathrm{A}^{\mathcal{C}}\left(\mathbf{a}_{\mid j}\right)$ calculated as follows

$$
\mathrm{A}^{\mathcal{C}}\left(\mathbf{a}_{\mid j}\right)=\sum_{k=1}^{j} C^{k}\left(\mathbf{a}_{\mid k}\right) .
$$

$\mathcal{C}$ is a conservative charge system if for all $j \in N$ and for each pair $\mathbf{a}, \mathbf{b} \in \mathcal{E}_{N^{\prime}}$, with $\Pi\left(\mathbf{a}_{\mid j}\right)=\Pi\left(\mathbf{b}_{\mid j}\right)$ we have that $\mathrm{A}^{\mathcal{C}}\left(\mathbf{a}_{\mid j}\right)=\mathrm{A}^{\mathcal{C}}\left(\mathbf{b}_{\mid j}\right)$.

Remark 2.1 Let $\sigma \in \Sigma_{E_{N^{\prime}}}$ be such that $w \in K^{\sigma}$ and let $\Gamma^{*} \in \mathcal{S P}^{N^{\prime}}$ be a most in $w$. We denote by $\mathbf{a}^{\Gamma^{*}, \sigma} \in \mathcal{E}_{N^{\prime}}^{\sigma}$ the unique vector such that 
$\left\{a_{1}^{\Gamma^{*}, \sigma}, a_{2}^{\Gamma, \sigma}, \ldots, a_{n}^{\Gamma^{*}, \sigma}\right\} \equiv \Gamma^{*}$. In addition, the allocation protocol $\kappa^{\mathcal{C}, \sigma}\left(\cdot, \mathbf{a}^{\Gamma^{*}, \sigma}\right)$, simply denoted as the map $\chi^{\mathcal{C}, \sigma}: K^{\sigma} \rightarrow \mathbb{R}^{N}$, is the Construct and Charge $(C C-)$ rule w.r.t. $\mathcal{C}$ and $\sigma$ introduced in Moretti et al. (2008).

Remark 2.2 Let $\sigma, \sigma^{\prime} \in \Sigma_{E_{N^{\prime}}}$ and let $K^{\sigma}$ and $K^{\sigma^{\prime}}$ be the respective Kruskal cones. Let $w \in K^{\sigma} \cap K^{\sigma^{\prime}}$. Moretti et al. (2008) proved that if $\mathcal{C}$ is conservative, then $\chi^{\mathcal{C}, \sigma}(w)=\chi^{\mathcal{C}, \sigma^{\prime}}(w)$.

\section{Minimum interval cost spanning trees}

This work deals with minimum interval cost spanning tree (micst) situations, i.e. situations where a set $N=\{1, \ldots, n\}$ of agents is willing to be connected as cheap as possible to a source (i.e. a supplier of a service) denoted by 0 , based on an uncertain weight (or cost) system of connection. Let $I\left(\mathbb{R}_{+}\right)$be the set of all closed intervals in $\mathbb{R}$. In the sequel we use also the notation $W$ for the interval weight function, i.e. a map which assigns to each edge $e \in E_{N^{\prime}}$ a closed interval $W(e) \in I\left(\mathbb{R}_{+}\right)$representing all possible uncertain weights or costs of edge $e$. The interval cost $W(e)$ of each edge $e \in E_{N^{\prime}}$ will be denoted by $[\underline{W}(e), \bar{W}(e)]$. No probability distribution is assumed for edge costs. We will denoted by $w^{s}(e) \in[\underline{W}(e), \bar{W}(e)]$ the realization of cost of edge $e \in E_{N^{\prime}}$ in scenario $s$. We will denote by $\mathcal{S}$ the set of all possible scenarios.

We denote an micst situation with set of users $N$, source 0 , and interval weight function $W$ by $\left\langle N^{\prime}, W\right\rangle$ (or simply $W$ ). Further, we denote by $\mathcal{I} \mathcal{W}^{N^{\prime}}$ the set of all micst situations $\left\langle N^{\prime}, W\right\rangle($ or $W)$ with node set $N^{\prime}$.

Example 3.1 We consider a micst situation with three agents denoted by 1, 2, and 3 and the source 0 . As depicted in Figure 1, to each edge $e \in E_{\{0,1,2,3\}}$ is assigned a closed interval $W(e) \in I\left(\mathbb{R}_{+}\right)$representing the uncertain cost of edge $e$. For each scenario $s \in \mathcal{S}$ we have a realization $w^{s} \in \mathcal{W}^{N^{\prime}}$ such that $w^{s}(\{0,1\}) \in[1,9], w^{s}(\{0,2\}) \in[6,11], w^{s}(\{0,3\}) \in[1,5], w^{s}(\{1,2\}) \in[2,4]$, $w^{s}(\{1,3\}) \in[1,3]$, and $w^{s}(\{2,3\}) \in[0,6]$. 


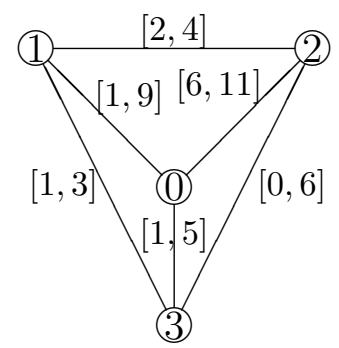

Figure 1: An micst situation $<\{0,1,2,3\}, W>$.

After the decision on which spanning tree must be constructed, agents face the problem of how to divide the cost of the spanning tree. In the following, we study this problem using the notion of cooperative cost game.

A cooperative cost game or cost game is a pair $(N, c)$, where $N$ denotes the finite set of players and $c: 2^{N} \rightarrow \mathbb{R}$ is the characteristic function, with $c(\emptyset)=0$ (here $2^{N}$ denotes the power set of player set $N$ ). Often we identify a cost game $(N, c)$ with the corresponding characteristic function $c$. A group of players $T \subseteq N$ is called a coalition and $c(T)$ is called the cost of this coalition. A payoff vector (or cost allocation) is a vector in $\mathbb{R}^{N}$.

A particular set, possibly empty, of cost allocations of a cost game $(N, c)$ is the core of $c$, which is defined as follows:

$$
\operatorname{Core}(c)=\left\{x \in \mathbb{R}^{N} \mid \sum_{i \in S} x_{i} \leq c(S) \forall S \in 2^{N} \backslash\{\emptyset\} ; \sum_{i \in N} x_{i}=c(N)\right\} .
$$

We first note that to each possible scenario $s \in \mathcal{S}$ with the associated realization $w^{s} \in \mathcal{W}^{N^{\prime}}$, corresponds to a mest game $\left(N, c_{w}\right)$ (or simply $c_{w^{s}}$ ), which is defined by

$$
c_{w^{s}}(T)=\min \left\{w^{s}(\Gamma) \mid \Gamma \text { is a spanning network on } T^{\prime}\right\}
$$

for every $T \in 2^{N} \backslash\{\emptyset\}$, with the convention that $c_{w}(\emptyset)=0$.

We call a map $F: \mathcal{W}^{N^{\prime}} \rightarrow \mathbb{R}^{N}$ assigning to every mcst situation $w$ a unique cost allocation in $\mathbb{R}^{N}$ a solution. A solution $F$ is a cost monotonic 
solution if for all mest situations $w, w^{\prime} \in \mathcal{W}^{N^{\prime}}$ such that $w(\bar{e}) \leq w^{\prime}(\bar{e})$ for one edge $\bar{e} \in E_{N^{\prime}}$ and $w(e)=w^{\prime}(e)$ for each $e \in E_{N^{\prime}} \backslash\{\bar{e}\}$, it holds that $F(w) \leq F\left(w^{\prime}\right)$.

All these notions may be useful to solve the cost allocation problem related to each scenario $s \in \mathcal{S}$, i.e. considering the associated realization $w^{s} \in \mathcal{W}^{N^{\prime}}$ and the corresponding mest game $c_{w^{s}}$.

We notice that, in many real cases, once a spanning tree $\Gamma$ is constructed under a scenario $s \in \mathcal{S}$, all that the agents know about $w^{s}$ is just the cost of $w^{s}(e)$, for each edge $e \in \Gamma$. However, in other realistic situations, only the total cost $w^{s}(\Gamma)$ is known. In all these circumstances, only few values of $c_{w^{s}}$ are revealed after the construction of a spanning tree $\Gamma$.

In following sections we present some criteria useful to solve the decision making problem on which spanning tree must be constructed in micst situations. For each criterium, we study some properties of cost allocation protocols based on charge systems.

\section{Permanent trees}

According to Yaman et al. (1999), a permanent tree in a micst situation $W \in \mathcal{I}^{\mathcal{W}^{N^{\prime}}}$ is a spanning tree $\Gamma^{P}$ which has minimum cost for each scenario $s \in \mathcal{S}$. In formula, a spanning tree $\Gamma^{P}$ is a permanent tree iff

$$
w^{s}\left(\Gamma^{P}\right) \leq w^{s}(\Gamma)
$$

for each scenario $s \in \mathcal{S}$ and each spanning tree $\Gamma$ in $w^{s}$.

Proposition 4.1 Let $W \in \mathcal{I W}^{N^{\prime}}$ and let $\Gamma^{P}$ be a permanent tree in $W$ and let $\mathcal{C}=\left\{C^{1}, \ldots, C^{n}\right\}$ be a conservative charge system on $N$. Then, the following assertions hold:

(i) $\kappa^{\mathcal{C}, \sigma}\left(w^{s}, \mathbf{a}^{\Gamma^{P}, \sigma}\right)=\chi^{\mathcal{C}, \sigma}\left(w^{s}\right)$ for each $s \in \mathcal{S}$ and each $\sigma \in \Sigma_{E_{N^{\prime}}}$ such that $w^{s} \in K^{\sigma}$ 
(ii) $\chi^{\mathcal{C}}\left(w^{s}\right)=\chi^{\mathcal{C}, \sigma}\left(w^{s}\right)=\chi^{\mathcal{C}, \sigma^{\prime}}\left(w^{s}\right) \in \operatorname{Core}\left(c_{w^{s}}\right)$ for each $s \in S$ and each $\sigma, \sigma^{\prime} \in \Sigma_{E_{N^{\prime}}}$ such that $w^{s} \in K^{\sigma} \cap K^{\sigma^{\prime}}$;

(iii) $\chi_{i}^{\mathcal{C}}\left(w^{s}\right) \leq \kappa_{i}^{\mathcal{C}, \sigma}\left(w^{s}, \mathbf{a}^{\Gamma, \sigma}\right)$ for all $i \in N, \Gamma \in \mathcal{S T}^{N^{\prime}}, s \in \mathcal{S}$ and each $\sigma \in \Sigma_{E_{N^{\prime}}}$ such that $w^{s} \in K^{\sigma}$.

Proof Assertion (i) directly follows by Remark 2.1 and relation (3), which means that $\Gamma^{P}$ is a mest in $w^{s}$, for each $s \in \mathcal{S}$.

Equalities in assertion (ii) follow by Remark 2.2. Moretti et al. (2008) showed that an allocation provided by a conservative $C C$-rule in a mcst situation $w \in \mathcal{W}^{N^{\prime}}$ belongs to the core of the game $c_{w}$. So, assertion (ii) is proved.

Now, to prove (iii), let $\Gamma \in \mathcal{S} \mathcal{T}^{N^{\prime}}, s \in \mathcal{S}$ and $\sigma \in \Sigma_{E_{N^{\prime}}}$ be such that $w^{s} \in K^{\sigma}$. Consider a mest situation $\tilde{w} \in \mathcal{W}^{N^{\prime}}$ such that

$$
\tilde{w}(e)= \begin{cases}w^{s}(e) & \text { if } e \in \Gamma \\ \max _{a \in E_{N^{\prime}}} w^{s}(a) & \text { otherwise. }\end{cases}
$$

Note that,

$$
w^{s}(e) \leq \tilde{w}(e)
$$

for each $e \in E_{N^{\prime}}$. Moreover, $\Gamma$ is a most in $\tilde{w}$.

Let $\tilde{\sigma} \in \Sigma_{E_{N^{\prime}}}$ be such that $\tilde{w} \in K^{\tilde{\sigma}}$. By Remark 2.1 we have

$$
\kappa^{\mathcal{C}, \tilde{\sigma}}\left(\tilde{w}, \mathbf{a}^{\Gamma, \tilde{\sigma}}\right)=\chi^{\mathcal{C}}(\tilde{w}) .
$$

Note that, by relation (4), we have $a_{i}^{\Gamma, \sigma}=a_{i}^{\Gamma, \tilde{\sigma}}$ for each $i \in N$. Then, by relation (1) we have

$$
\kappa^{\mathcal{C}, \tilde{\sigma}}\left(\tilde{w}, \mathbf{a}^{\Gamma, \tilde{\sigma}}\right)=\sum_{i=1}^{n} \tilde{w}\left(a_{i}^{\Gamma}\right) C^{i}=\sum_{i=1}^{n} w^{s}\left(a_{i}^{\Gamma}\right) C^{i}=\kappa^{\mathcal{C}, \sigma}\left(w^{s}, \mathbf{a}^{\Gamma, \sigma}\right) .
$$

Finally, we have that for each $i \in N$

$$
\chi_{i}^{\mathcal{C}}\left(w^{s}\right) \leq \chi_{i}^{\mathcal{C}}(\tilde{w})=\kappa_{i}^{\mathcal{C}, \tilde{\sigma}}\left(\tilde{w}, \mathbf{a}^{\Gamma, \tilde{\sigma}}\right)=\kappa_{i}^{\mathcal{C}, \sigma}\left(w^{s}, \mathbf{a}^{\Gamma, \sigma}\right)
$$

where the inequality follows from relation (5) and the fact that conservative $C C$-rules are cost monotonic (see Moretti et al. (2008), Tijs et al. (2006)), 
and the equalities follow from relations (6) and (7), respectively.

For each $W \in \mathcal{I W}^{N^{\prime}}$, Yaman et al. (1999) proved that a spanning tree $\Gamma \in \mathcal{S} \mathcal{T}^{N^{\prime}}$ in $W$ is a permanent tree if and only if $\Gamma$ is a mest under a scenario $\breve{s} \in \mathcal{S}$ such that $w^{\breve{s}}(e)=\bar{W}(e)$ for each $e \in \Gamma$ and $w^{\breve{s}}(e)=\underline{W}(e)$ for each $e \in E_{N^{\prime}} \backslash \Gamma$. We use this result to provide an example where a special conservative Construct and Charge rule, the $P$-value (Branzei et al. (2004)), is used to share the cost of a permanent tree among agents.

Example 4.1 Consider the set of functions $\hat{\mathcal{C}}=\left\{\hat{C}^{1}, \ldots, \hat{C}^{n}\right\}$ on $N$ such that for each $\boldsymbol{a}=\left(a_{1}, \ldots, a_{n}\right) \in \mathcal{E}_{N^{\prime}}$ and for each $j \in N$

$$
\hat{C}_{i}^{j}\left(\boldsymbol{a}_{\mid j}\right)= \begin{cases}\frac{1}{\left|S\left(\Pi\left(\boldsymbol{a}_{\mid j-1}\right),\{i\}\right)\right|}-\frac{1}{\left|S\left(\Pi\left(\boldsymbol{a}_{\mid j}\right),\{i\}\right)\right|} & \text { if } 0 \notin S\left(\Pi\left(\boldsymbol{a}_{\mid j}\right), a_{j}\right) \\ \frac{1}{\left|S\left(\Pi\left(\boldsymbol{a}_{\mid j-1}\right),\{i\}\right)\right|} & \text { if }\{0, i\} \subseteq S\left(\Pi\left(\boldsymbol{a}_{\mid j}\right), a_{j}\right) \\ & \text { and } 0 \notin S\left(\Pi\left(\boldsymbol{a}_{\mid j-1}\right),\{i\}\right), \\ 0 & \text { otherwise, }\end{cases}
$$

for each $i \in N$. Moretti et al. (2008) proved that $\hat{\mathcal{C}}$ is a conservative charge system. They also proved that the conservative $C C$-rule $\chi^{\hat{\mathcal{C}}}$ equals the $P$ value for mcst situations (Branzei et al. (2004), Feltkamp et al. (1994), Bergañtinos and Vidal-Puga (2007)).

Consider the micst situation $<\{0,1,2,3\}, W>$ depicted in Figure 2. The unique permanent tree in $W$ is $\Gamma^{P}=\{\{0,1\},\{0,3\},\{1,2\}\}$. Let $s \in \mathcal{S}$ and let $\sigma \in \Sigma_{E_{N^{\prime}}}$ be such that $w^{s} \in K^{\sigma}$. By Proposition 4.1 we have that $\chi^{\hat{\mathcal{C}}}\left(w^{s}\right)=\kappa^{\hat{\mathcal{C}}, \sigma}\left(w^{s}, \mathbf{a}^{\Gamma^{P}, \sigma}\right)$. More precisely, we have that $\chi_{1}^{\hat{\mathcal{C}}}\left(w^{s}\right)=w^{s}(\{0,1\})$, if $w^{s}(\{0,1\}) \leq w^{s}(\{1,3\})$, and $\chi_{1}^{\hat{\mathcal{C}}}\left(w^{s}\right)=\frac{1}{2} w^{s}(\{0,1\})+\frac{1}{2} w^{s}(\{1,3\})$, otherwise; $\chi_{3}^{\hat{\mathcal{C}}}\left(w^{s}\right)=w^{s}(\{0,1\})+w^{s}(\{1,3\})-\chi_{1}^{\hat{\mathcal{C}}}\left(w^{s}\right) ; \chi_{2}^{\hat{\mathcal{C}}}\left(w^{s}\right)=w^{s}(\{1,2\})$. 


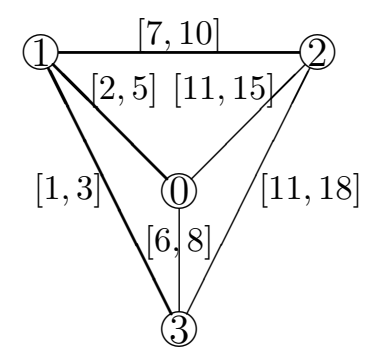

Figure 2: An micst situation $<\{0,1,2,3\}, W>$ and the related permanent most (thicker lines).

\section{A pessimistic approach}

Given a micst situation $W \in \mathcal{I W}^{N^{\prime}}$ and a spanning tree $\Gamma$ in $W$, an absolute worst case scenario $s_{\Gamma}^{*}$ for $\Gamma$ is a scenario in which the cost of $\Gamma$ is the maximum. In formula, $s_{\Gamma}^{*} \in \arg \max _{s \in S} w^{s}(\Gamma)$. It follows from this definition that in an absolute worst case scenario for a given spanning tree $\Gamma$ the costs of all edges $e \in \Gamma$ are fixed at their upper bounds and the costs of the remaining edges can assume any value in their intervals.

A spanning tree $\Gamma^{A}$ in $W$ whose cost is the minimum under absolute worst case scenarios is called, according to Yaman et al. (1999, 2001), absolute robust spanning tree in $W$, i.e. $\Gamma^{A} \in \arg \min _{\Gamma \in \mathcal{S} \mathcal{T}^{N^{\prime}}} \max _{s \in S} w^{s}(\Gamma)$. In order to study the cost allocation problem arising from a micst situation $W \in \mathcal{I W}^{N^{\prime}}$, we introduce the notion of pessimistic spanning tree game $\left(N, c_{W}^{A}\right)$ (or simply $\left.c_{W}^{A}\right)$, which is defined by

$$
c_{W}^{A}(T)=\min _{\Gamma \in \mathcal{S} \mathcal{T}^{T^{\prime}}}\left\{w^{s_{\Gamma}^{*}}(\Gamma) \mid s_{\Gamma}^{*} \text { is an absolute worst case scenario for } \Gamma\right\}
$$

for every $T \in 2^{N} \backslash\{\emptyset\}$ with the convention that $c_{W}^{A}(\emptyset)=0$. The following proposition provides an interesting property of allocation protocols based on charge systems when the pessimistic spanning tree game is considered.

Proposition 5.1 Let $W \in \mathcal{I W}^{N^{\prime}}$, let $\Gamma^{A}$ be an absolute robust spanning tree in $W$ and let $s_{\Gamma^{A}}^{*}$ be an absolute worst case scenario for $\Gamma^{A}$. Let $\mathcal{C}=$ $\left\{C^{1}, \ldots, C^{n}\right\}$ be a charge system on $N$ and let $\sigma \in \Sigma_{E_{N^{\prime}}}$ be such that $w^{s^{*} A} \in$ 
$K^{\sigma}$. Then,

$$
\kappa^{\mathcal{C}, \sigma}\left(w^{s_{\Gamma}^{*}}, \mathbf{a}^{\Gamma^{A}, \sigma}\right)=\chi^{\mathcal{C}}\left(w^{s^{*} A}\right) \in \operatorname{Core}\left(c_{W}^{A}\right) .
$$

Proof Consider the scenario in which all edge costs are at their upper bounds. The set of minimum spanning trees under this scenario is exactly the set of absolute robust spanning trees (see e.g. Yaman et al. (1999)). Then $\Gamma^{A}$ is a mcst for $w^{s^{*} A} \in \mathcal{W}^{N^{\prime}}$, where $w^{s^{*} A}(e)=\bar{W}(e)$ for each edge $e \in E_{N^{\prime}}$. Then, by Remark 2.1 we have that $\kappa^{\mathcal{C}, \sigma}\left(w^{s^{*}} \Gamma^{A}, \mathbf{a}^{\Gamma^{A}, \sigma}\right)$ equals the $C C$-rule $\chi^{\mathcal{C}}\left(w^{s^{*} A}\right)$.

We conclude the proof with the remark that the allocation provided by a conservative $C C$-rule in a mcst situation $w \in \mathcal{W}^{N^{\prime}}$ belongs to the core of the game $c_{w}$ (Moretti et al. (2008)).

According to this pessimistic approach, it is also reasonable to expect that each agent $i \in N$ would support the construction of a spanning tree $\Gamma$ which makes minimum the cost allocated to agent $i$ in an absolute worst case scenario. Next proposition shows that if conservative $C C$-rules are adopted, then such a tree $\Gamma$ coincides with an absolute robust spanning tree $\Gamma^{A}$.

Proposition 5.2 Let $W \in \mathcal{I W}^{N^{\prime}}$ and let $\Gamma^{A}$ be an absolute robust spanning tree in $W$ and let $s_{\Gamma^{A}}^{*}$ be an absolute worst case scenario for $\Gamma^{A}$. Let $\mathcal{C}=$ $\left\{C^{1}, \ldots, C^{n}\right\}$ be a conservative charge system on $N$ and let $\sigma \in \Sigma_{E_{N^{\prime}}}$ be such that $w^{s^{*}}{ }^{A} \in K^{\sigma}$. Then,

$$
\kappa_{i}^{\mathcal{C}, \sigma^{\prime}}\left(w^{s_{\Gamma}^{*}}, a^{\Gamma, \sigma^{\prime}}\right) \geq \kappa_{i}^{\mathcal{C}, \sigma^{\prime}}\left(w^{s_{\Gamma}^{*} A}, a^{\Gamma^{A}, \sigma}\right)
$$

for all $i \in N$ and $\Gamma \in \mathcal{S T}^{N^{\prime}}$, where $s_{\Gamma}^{*}$ is an absolute worst case scenario for $\Gamma$ and $\sigma^{\prime} \in \Sigma_{E_{N^{\prime}}}$ is such that $w^{s_{\Gamma}^{*}} \in K^{\sigma^{\prime}}$.

Proof Let $\Gamma \in \mathcal{S} \mathcal{T}^{N^{\prime}}$ be such that $\Gamma \neq \Gamma^{A}$ and let $s_{\Gamma}^{*} \in \mathcal{S}$ be an absolute worst case scenario for $\Gamma$, in formula

$$
w^{s^{*}}(\Gamma)=\max _{s \in \mathcal{S}} w^{s}(\Gamma) .
$$

By relation (11) it follows that

$$
w^{s^{*} A}\left(a_{i}^{\Gamma, \hat{\sigma}}\right) \leq w^{s_{\Gamma}^{*}}\left(a_{i}^{\Gamma, \hat{\sigma}}\right)
$$


for each $i \in N$, where $\sigma^{\prime} \in \Sigma_{E_{N^{\prime}}}$ is such that $w^{s_{\Gamma}^{*}} \in K^{\sigma^{\prime}}$.

Recall that by Remark 2.1

$$
\kappa^{\mathcal{C}, \sigma^{\prime}}\left(w^{s_{\Gamma}^{*}}, a^{\Gamma, \sigma^{\prime}}\right)=\chi^{\mathcal{C}}\left(w^{s_{\Gamma}^{*}}\right)
$$

and

$$
\kappa^{\mathcal{C}, \sigma^{\prime}}\left(w^{s^{*}{ }^{A}}, a^{\Gamma^{A}, \sigma}\right)=\chi^{\mathcal{C}}\left(w^{s^{*} \Gamma^{A}}\right) .
$$

Now define a mcst situation $\tilde{w} \in \mathcal{W}^{N^{\prime}}$ as follows

$$
\tilde{w}(e)= \begin{cases}w^{s_{\Gamma}^{*}}(e) & \text { if } e \in \Gamma \\ \max _{a \in E_{N^{\prime}}} w^{s^{*}} \Gamma^{A}(a) & \text { otherwise. }\end{cases}
$$

By cost monotonicity of $\chi^{\mathcal{C}}$ we have that

$$
\chi_{i}^{\mathcal{C}}(\tilde{w}) \geq \chi_{i}^{\mathcal{C}}\left(w^{s^{*} \Gamma^{A}}\right)
$$

for each $i \in N$.

By relation (13), (15) and (1) we have that

$$
\chi_{i}^{\mathcal{C}}\left(w^{s_{\Gamma}^{*}}\right)=\sum_{i=1}^{n} w_{\Gamma}^{s_{\Gamma}^{*}}\left(a_{i}^{\Gamma}\right) C^{i}=\chi_{i}^{\mathcal{C}}(\tilde{w})
$$

Finally, by relations (13), (14), (16) and (17) we have

$$
\kappa^{\mathcal{C}, \sigma^{\prime}}\left(w^{s_{\Gamma}^{*}}, a^{\Gamma, \sigma^{\prime}}\right)=\chi_{i}^{\mathcal{C}}\left(w^{s_{\Gamma}^{*}}\right) \geq \chi_{i}^{\mathcal{C}}\left(w^{s^{*} A}\right)=\kappa^{\mathcal{C}, \sigma^{\prime}}\left(w^{s^{*} A}, a^{\Gamma^{A}, \sigma}\right) .
$$

Suppose that there is agreement among the players about the allocation protocol to be used. The property for allocation protocols based on conservative charge systems which is stated by Proposition 5.2, captures a condition for the "stability" of the decision to construct an absolute robust spanning tree: no group of pessimistic agents has any incentive to move from an absolute robust spanning tree to another spanning tree. The following example illustrates this property on a particular instance. 
Example 5.1 Consider the micst situation $<\{0,1,2,3\}, W>$ depicted in Figure 1. Note that in this micst situation $W$ there are no permanent trees.

The unique absolute robust spanning tree in $W$ is $\Gamma^{A}=\{\{0,3\},\{1,3\}$, $\{1,2\}\}$. Let $s_{\Gamma^{A}}^{*}$ be an absolute worst case scenario for $\Gamma^{A}$. Then, $w^{s^{*}}=$ $\bar{W}(e)$ for each $e \in E_{N^{\prime}}$. The corresponding pessimistic spanning tree game $c_{W}^{A}$ is given in Table 2.

\begin{tabular}{|r||r|r|r|r|r|r|r|}
\hline$T$ & $\{1\}$ & $\{2\}$ & $\{3\}$ & $\{1,2\}$ & $\{1,3\}$ & $\{2,3\}$ & $\{1,2,3\}$ \\
\hline \hline$c_{W}^{A}(T)$ & 9 & 11 & 5 & 13 & 8 & 11 & 12 \\
\hline
\end{tabular}

Table 1: Pessimistic spanning tree game $c_{W}^{A}$ corresponding to the micst situation $W$ depicted in Figure 1.

Consider again the conservative charge system of Example 4.1 and the related conservative $C C$-rule $\chi^{\hat{\mathcal{C}}}$ and let $\sigma \in \Sigma_{E_{N^{\prime}}}$ be such that $w^{s^{*}{ }^{*}} \in K^{\sigma}$. Then, $\kappa_{1}^{\hat{\mathcal{C}}, \sigma}\left(w^{s^{*} A}, \mathbf{a}^{\Gamma^{A}, \sigma}\right)=\chi_{1}^{\hat{\mathcal{C}}}\left(w^{s^{*} \Gamma^{A}}\right)=\frac{1}{2} 3+\frac{1}{6} 4+\frac{1}{3} 5=\frac{23}{6} ; \kappa_{2}^{\hat{\mathcal{C}}, \sigma}\left(w^{s^{*} A}, \mathbf{a}^{\Gamma^{A}, \sigma}\right)=$ $\chi_{2}^{\hat{\mathcal{C}}}\left(w^{s^{*}} \Gamma^{A}\right)=\frac{2}{3} 4+\frac{1}{3} 5=\frac{26}{6} ; \kappa_{3}^{\hat{\mathcal{C}}, \sigma}\left(w^{s^{*}}{ }^{*}, \mathbf{a}^{\Gamma^{A}, \sigma}\right)=\chi_{3}^{\hat{\mathcal{C}}}\left(w^{s^{*}}{ }^{*}\right)=\frac{1}{2} 3+\frac{1}{6} 4+\frac{1}{3} 5=\frac{23}{6}$.

According to Proposition 5.2, there are no $\Gamma \in \mathcal{S T}^{N^{\prime}}$ and $i \in N$ such that $\kappa_{i}^{\hat{\mathcal{C}}, \sigma}\left(w_{\Gamma}^{s_{\Gamma}^{*}}, \mathbf{a}^{\Gamma^{A}, \sigma}\right)>\kappa_{i}^{\hat{\mathcal{C}}, \sigma^{\prime}}\left(w^{s_{\Gamma}^{*}}, \mathbf{a}^{\Gamma, \sigma^{\prime}}\right)$, where $s_{\Gamma}^{*}$ is an absolute worst case scenario for $\Gamma$ and $\sigma^{\prime} \in \Sigma_{E_{N^{\prime}}}$ is such that $w^{s_{\Gamma}^{*}} \in K^{\sigma^{\prime}}$.

\section{An optimistic approach}

Following a similar line as in Section 5, given a micst situation $W \in \mathcal{I W}^{N^{\prime}}$ and a spanning tree $\Gamma$ in $W$, we define an absolute best case scenario $s_{\Gamma}^{\circ}$ for $\Gamma$, as a scenario in which the cost of $\Gamma$ is the minimum. In formula, $s_{\Gamma}^{\circ} \in \arg \min _{s \in S} w^{s}(\Gamma)$. A spanning tree in $W$ whose cost is the minimum under the absolute best case scenarios is denoted by $\Gamma^{B}$, i.e. $\Gamma^{B} \in$ $\arg \min _{\Gamma \in \mathcal{S} \mathcal{T}^{N^{\prime}}} \min _{s \in S} w^{s}(\Gamma)$.

For each micst situation $W \in \mathcal{I} \mathcal{W}^{N^{\prime}}$, we may define the optimistic span- 
ning tree game $\left(N, c_{W}^{B}\right)$ (or simply $c_{W}^{B}$ ), which is defined by

$$
c_{W}^{B}(S)=\min _{\Gamma \in \mathcal{S} \mathcal{T}^{T^{\prime}}}\left\{w^{s_{\Gamma}^{\circ}}(\Gamma) \mid s_{\Gamma}^{\circ} \text { is an absolute best case scenario for } \Gamma\right\}
$$

for every $S \in 2^{N} \backslash\{\emptyset\}$ with the convention that $c_{W}^{B}(\emptyset)=0$.

Proposition 6.1 Let $W \in \mathcal{I W}^{N^{\prime}}$, let $\Gamma^{B} \in \arg \min _{\Gamma \in \mathcal{S} \mathcal{T}^{N^{\prime}}} \min _{s \in S} w^{s}(\Gamma)$ and let $s_{\Gamma^{B}}^{\circ}$ be an absolute best case scenario for $\Gamma^{B}$. Let $\mathcal{C}=\left\{C^{1}, \ldots, C^{n}\right\}$ be a charge system on $N$ and let $\sigma \in \Sigma_{E_{N^{\prime}}}$ be such that $w^{s_{\Gamma}^{\circ}} \in K^{\sigma}$. Then,

$$
\kappa_{i}^{\mathcal{C}, \sigma}\left(w^{s^{\circ} \Gamma^{\circ}}, \mathbf{a}^{\Gamma^{B}, \sigma}\right) \in \operatorname{Core}\left(c_{W}^{B}\right) .
$$

Proof Consider the scenario in which all edge costs are at their lower bounds. The set of minimum spanning trees under this scenario is exactly the set of spanning trees whose cost is the minimum under the absolute best case scenarios. Then $\Gamma^{B}$ is a mest for $w^{s^{\circ}} \in \mathcal{W}^{N^{\prime}}$, where $w^{s^{\circ}}{ }^{\circ}(e)=\underline{W}(e)$ for each edge $e \in E_{N^{\prime}}$. The proof follows as the proof of Proposition 5.1.

Similar to Proposition 5.2, we may claim the following proposition.

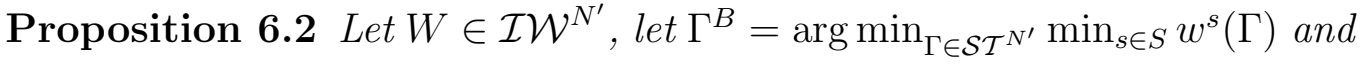
let $s_{\Gamma^{B}}^{\circ}$ be an absolute best case scenario for $\Gamma^{B}$. Let $\mathcal{C}=\left\{C^{1}, \ldots, C^{n}\right\}$ be a conservative charge system on $N$ and let $\sigma \in \Sigma_{E_{N^{\prime}}}$ be such that $w^{s^{\circ}} \in K^{\sigma}$. Then,

$$
\kappa_{i}^{\mathcal{C}, \sigma^{\prime}}\left(w^{s_{\Gamma}^{\circ}}, a^{\Gamma, \sigma^{\prime}}\right) \geq \kappa_{i}^{\mathcal{C}, \sigma^{\prime}}\left(w^{s_{\Gamma}^{\circ}}, a^{\Gamma^{B}, \sigma}\right)
$$

for all $i \in N$ and each $\Gamma \in \mathcal{S} \mathcal{T}^{N^{\prime}}$, where $s_{\Gamma}^{\circ}$ is an absolute best case scenario for $\Gamma$ and $\sigma^{\prime} \in \Sigma_{E_{N^{\prime}}}$ is such that $w^{s_{\Gamma}^{\circ}} \in K^{\sigma^{\prime}}$.

Proof Similar to the proof of Proposition 5.2.

Example 6.1 Consider the micst situation $<\{0,1,2,3\}, W>$ depicted in Figure 1. 


\begin{tabular}{|r||r|r|r|r|r|r|r|}
\hline$T$ & $\{1\}$ & $\{2\}$ & $\{3\}$ & $\{1,2\}$ & $\{1,3\}$ & $\{2,3\}$ & $\{1,2,3\}$ \\
\hline \hline$c_{W}^{B}(T)$ & 1 & 6 & 1 & 3 & 2 & 1 & 2 \\
\hline
\end{tabular}

Table 2: Optimistic spanning tree game $c_{W}^{B}$ corresponding to the micst situation $W$ depicted in Figure 1.

Let $\Gamma^{B}=\{\{0,1\},\{0,3\},\{2,3\}\}$ and let $s_{\Gamma^{B}}^{\circ}$ be an absolute best case scenario for $\Gamma^{B}$. Note that $w^{s^{\circ}}\left(\Gamma^{B}\right)=\min _{\Gamma \in \mathcal{S} \mathcal{T}^{N^{\prime}}} \min _{s \in S} w^{s}(\Gamma)=2$. The corresponding optimistic spanning tree game $c_{W}^{B}$ is given in Table 2.

Consider again the conservative charge system of Example 4.1 and the related conservative $C C$-rule $\chi^{\hat{\mathcal{C}}}$ and let $\sigma \in \Sigma_{E_{N^{\prime}}}$ be such that $w^{s^{\circ} B} \in K^{\sigma}$. Then, $\kappa_{1}^{\hat{\mathcal{C}}, \sigma}\left(w^{s^{\circ} B}, \mathbf{a}^{\Gamma^{B}, \sigma}\right)=\chi_{1}^{\hat{\mathcal{C}}}\left(w^{s^{\circ} B}\right)=1 ; \kappa_{2}^{\hat{\mathcal{C}}, \sigma}\left(w^{s^{\circ} B}, \mathbf{a}^{\Gamma^{B}, \sigma}\right)=\chi_{2}^{\hat{\mathcal{C}}}\left(w^{s^{\circ} B}\right)=\frac{1}{2} ;$ $\kappa_{3}^{\hat{\mathcal{C}}, \sigma}\left(w^{s^{\circ}}, \mathbf{a}^{\Gamma^{B}, \sigma}\right)=\chi_{3}^{\hat{\mathcal{C}}}\left(w^{s_{\Gamma}^{\circ}}\right)=\frac{1}{2}$.

According to Proposition 5.2, there are no $\Gamma \in \mathcal{S T}^{N^{\prime}}$ and $i \in N$ such that $\kappa_{i}^{\hat{\mathcal{C}}, \sigma}\left(w^{s_{\Gamma}^{\circ}}, \mathbf{a}^{\Gamma^{B}, \sigma}\right)>\kappa_{i}^{\hat{\mathcal{C}}, \sigma^{\prime}}\left(w^{s_{\Gamma}^{\circ}}, \mathbf{a}^{\Gamma, \sigma^{\prime}}\right)$, where $s_{\Gamma}^{\circ}$ is an absolute best case scenario for $\Gamma$ and $\sigma^{\prime} \in \Sigma_{E_{N^{\prime}}}$ is such that $w^{s_{\Gamma}^{\circ}} \in K^{\sigma^{\prime}}$.

Remark 6.1 Let $W \in \mathcal{I}^{\mathcal{W}^{N^{\prime}}}$ be such that $\Gamma^{P}$ is a permanent tree in $W$. Then $\Gamma^{A}=\Gamma^{B}=\Gamma^{P}$.

\section{Cost allocation problem after realizations}

Let $W \in \mathcal{I W}^{N^{\prime}}$. If all the agents in $N$ decide to adopt the pessimistic approach, then they will construct the spanning tree $\Gamma^{A}$ in $W$. On the other hand, if they decide to adopt the optimistic approach they will construct the spanning tree $\Gamma^{B}$ in $W$. Using the pessimistic approach, if scenario $s \in \mathcal{S}$ is realized, then the saving $w^{s^{*}} \Gamma^{A}\left(\Gamma^{A}\right)-w^{s}\left(\Gamma^{A}\right) \geq 0$ should be redistributed among agents. Using the optimistic approach, if scenario $s \in \mathcal{S}$ is realized, then the extra cost $w^{s}\left(\Gamma^{B}\right)-w^{s^{\circ}}\left(\Gamma^{B}\right) \geq 0$ should be reallocated among agents.

Suppose that an allocation protocol based on a conservative charge system $\mathcal{C}=\left\{C^{1}, \ldots, C^{n}\right\}$ is used and let $\Gamma^{A}$ be the spanning tree which is 
constructed, according to the pessimistic approach, under scenario $s \in S$. We distinguish two cases:

1.A) the cost of $w^{s}(e)$ is known, for each $e \in \Gamma^{A}$. Then a reasonable way to share the saving $w^{s^{*}} \Gamma^{A}\left(\Gamma^{A}\right)-w^{s}\left(\Gamma^{A}\right) \geq 0$ is to give to each agent $i \in N$ the $\Delta_{i}^{A}$ saving

$$
\Delta_{i}^{A}=\kappa_{i}^{\mathcal{C}, \sigma}\left(w^{s^{*}}{ }^{A}, \mathbf{a}^{\Gamma^{A}, \sigma}\right)-\kappa_{i}^{\mathcal{C}, \sigma^{\prime}}\left(w^{s}, \mathbf{a}^{\Gamma^{A}, \sigma^{\prime}}\right)
$$

where $\sigma \in \Sigma_{E_{N^{\prime}}}$ is such that $w^{s^{*}} \Gamma^{A} \in K^{\sigma}$ and $\sigma^{\prime} \in \Sigma_{E_{N^{\prime}}}$ is such that $w^{s} \in K^{\sigma^{\prime}}$.

2.A) only the total cost $w^{s}\left(\Gamma^{A}\right)$ is known. Let $\sigma \in \Sigma_{E_{N^{\prime}}}$ be such that $w^{s^{*} \Gamma^{A}} \in K^{\sigma}$. In order to allocate the cost saving $w^{s^{*} A}\left(\Gamma^{A}\right)-w^{s}\left(\Gamma^{A}\right)$, one possibility is to divide it proportionally to previous agents' contributions $\kappa_{i}^{\mathcal{C}, \sigma}\left(w^{s^{*} \Gamma^{A}}, \mathbf{a}^{\Gamma^{A}, \sigma}\right)$, for each $i \in N$; in formula ${ }^{1}$

$$
\operatorname{PROP}_{i}^{A}=\frac{\kappa_{i}^{\mathcal{C}, \sigma}\left(w^{s_{\Gamma}^{*}}, \mathbf{a}^{\Gamma^{A}, \sigma}\right)}{\sum_{k=1}^{n} \kappa_{k}^{\mathcal{C}, \sigma}\left(w^{s^{*}} \Gamma^{A}, \mathbf{a}^{\Gamma^{A}, \sigma}\right)}\left(w^{s^{*}{ }^{A}}\left(\Gamma^{A}\right)-w^{s}\left(\Gamma^{A}\right)\right) .
$$

The taxation problem related to the optimistic approach, after the spanning tree $\Gamma^{B}$ is constructed, is very close to lines above. We distinguish again two cases:

1.B) the cost of $w^{s}(e)$ is known, for each $e \in \Gamma^{B}$. Then the extra cost to be divide now is $w^{s}\left(\Gamma^{B}\right)-w^{s^{\circ}}\left(\Gamma^{B}\right) \geq 0$ and to each agent $i \in N$ is allocated the $\Delta_{i}^{B}$ cost

$$
\Delta_{i}^{B}=\kappa_{i}^{\mathcal{C}, \sigma^{\prime}}\left(w^{s}, \mathbf{a}^{\Gamma^{B}, \sigma^{\prime}}\right)-\kappa_{i}^{\mathcal{C}, \sigma}\left(w^{s^{\circ} B}, \mathbf{a}^{\Gamma^{B}, \sigma}\right)
$$

where $\sigma \in \Sigma_{E_{N^{\prime}}}$ is such that $w^{s^{\circ} B} \in K^{\sigma}$ and $\sigma^{\prime} \in \Sigma_{E_{N^{\prime}}}$ is such that $w^{s} \in K^{\sigma^{\prime}}$.

\footnotetext{
${ }^{1}$ In this case it is possible to look at the saving allocation problem as a bankruptcy problem where each agent claims the share of savings necessary to null his/her previous contribution, i.e. consider the bankruptcy problem (Aumann and Maschler (1985)) $\left(w^{s_{\Gamma}^{*} A}\left(\Gamma^{A}\right)-w^{\mathcal{S}}\left(\Gamma^{A}\right) ; \kappa_{1}^{\mathcal{C}, \sigma}\left(w^{s_{\Gamma}^{*} A}, \mathbf{a}^{\Gamma^{A}, \sigma}\right), \ldots, \kappa_{n}^{\mathcal{C}, \sigma}\left(w^{s_{\Gamma}^{*} A}, \mathbf{a}^{\Gamma^{A}, \sigma}\right)\right)$. Note that allocation provided by relation (20) corresponds to the proportional rule (Curiel et al. (1987)) for bankruptcy problems.
} 
2.B) only the total cost $w^{s}\left(\Gamma^{B}\right)$ is known. Similar to point (2.A), in this case the extra cost $w^{s}\left(\Gamma^{B}\right)-w^{s^{\circ}}\left(\Gamma^{B}\right) \geq 0$ is divided proportionally according to the following formula

$$
P R O P_{i}^{B}=\frac{\kappa_{i}^{\mathcal{C}, \sigma}\left(w^{s^{\circ} \Gamma^{\circ}}, \mathbf{a}^{\Gamma^{B}, \sigma}\right)}{\sum_{k=1}^{n} \kappa_{k}^{\mathcal{C}, \sigma}\left(w^{s^{\circ}}, \mathbf{a}^{\Gamma^{B}, \sigma}\right)}\left(w^{s}\left(\Gamma^{B}\right)-w^{s_{\Gamma}^{\circ}}\left(\Gamma^{B}\right)\right),
$$

for each $i \in N$.

Example 7.1 Consider the micst situation $<\{0,1,2,3\}, W>$ depicted in Figure 1. Note that in this micst situation $W$ there are no permanent trees. Consider again the conservative charge system of Example 4.1 and the related conservative $C C$-rule $\chi^{\hat{\mathcal{C}}}$. Suppose that a scenario $s \in \mathcal{S}$ is realized and that the associated mcst situation $w^{s}$ is depicted in Figure 3. Note that $w^{s}\left(\Gamma^{A}\right)=9$ and $w^{s}\left(\Gamma^{B}\right)=10$. The allocations of the saving $w^{s^{*} A}\left(\Gamma^{A}\right)-w^{s}\left(\Gamma^{A}\right)=12-9=$

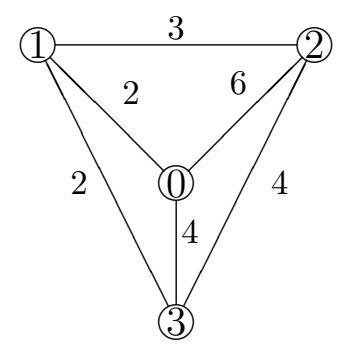

Figure 3: The mcst situation $<\{0,1,2,3\}, w^{s}>$ realized under scenario $s$.

3 computed according to (19) and (20) and the allocations of the extra cost $w^{s}\left(\Gamma^{B}\right)-w_{\Gamma^{B}}^{s^{\circ}}\left(\Gamma^{B}\right)=10-2=8$ computed according to (21) and (22) are shown in Table 3.

\section{Concluding remarks}

In this paper we cope with uncertainty in cost allocation problems arising from connection situations. Basically, we deal with mcst situations where costs are intervals and agents may act optimistically or pessimistically. 


\begin{tabular}{|c||c|c|c|}
\hline agent & 1 & 2 & 3 \\
\hline \hline$\Delta^{A}$ & $\frac{23}{6}-\left(\frac{1}{2} 2+\frac{1}{6} 3+\frac{1}{3} 4\right)=1$ & $\frac{26}{6}-\left(\frac{2}{3} 3+\frac{1}{3} 4\right)=1$ & $\frac{23}{6}-\left(\frac{1}{2} 2+\frac{1}{6} 3+\frac{1}{3} 4\right)=1$ \\
\hline$P R O P^{A}$ & $\frac{23}{12}(12-9)=\frac{23}{24}$ & $\frac{26}{12}(12-9)=\frac{26}{24}$ & $\frac{23}{12}(12-9)=\frac{23}{24}$ \\
\hline \hline$\Delta^{B}$ & $2-1=1$ & $4-\frac{1}{2}=\frac{7}{2}$ & $4-\frac{1}{2}=\frac{7}{2}$ \\
\hline$P R O P^{B}$ & $\frac{1}{2}(10-2)=4$ & $\frac{\frac{1}{2}}{2}(10-2)=2$ & $\frac{1}{2}(10-2)=2$ \\
\hline
\end{tabular}

Table 3: Allocations of savings $\left(\Delta^{A}\right.$ and $\left.P R O P^{A}\right)$ and of extra costs $\left(\Delta^{B}\right.$ and $P R O P^{B}$ ) after the realization of scenario $s$.

In the sequel, we briefly introduce a more complex problem as a possible topic for further research: how to deal with micst situations where not all the agents follow the same (pessimistic or optimistic) approach to make the decision on which spanning tree must be realized?

In theory of decisions under uncertainty, it is well known the Hurwicz's criterion (see Wald (1950)), giving to a decision of a single decision maker a value which is a weighted sum of the worst (pessimistic) and best (optimistic) possible outcomes, where the weight is represented by an $\alpha$ which is known as an index of pessimism / optimism. How to determine such an $\alpha$ index for a single decision maker in an uncertain situation is a difficult task. In micst situations, things are much more complicated by the presence of $n>$ 1 agents, and the related problem to find $n$, possibly different, indices of pessimism/optimism. In addition, even if such $\alpha_{1}, \ldots, \alpha_{n}$ are given, it is not clear how this information may help to chose and realize an optimal spanning tree.

We finally remark that, to take the decision on whether to cooperate or not, the pessimistic spanning tree game $c_{W}^{A}$ and the optimistic spanning tree game $c_{W}^{B}$ set the bounds of the cost games that would be interesting to consider in a micst situation $W \in \mathcal{I}^{\mathcal{W}^{N^{\prime}}}$ where agents have different levels of pessimism/optimism. These bounds may be given in terms of interval cost 
games (Branzei, et al. (2003), Alparslan Gök et al. (2008)), i.e. defining the corresponding micst game $c_{W}=\left[c_{W}^{A}, c_{W}^{B}\right]$. The model of cooperative interval games was firstly introduced in Branzei, et al. (2003) to model bankruptcy situations under interval uncertainty of claims. The theory of cooperative interval games has been recently developed. We refer here to Alparslan Gök et al. (2008), Alparslan Gök et al. (2008a; b; c; d).

\section{References}

Alparslan Gök S.Z., Miquel S., Tijs S. (2008) Cooperation under interval uncertainty. Preprint no. 73, Institute of Applied Mathematics, METU and Tilburg University, Center for Economic Research, The Netherlands, CentER DP 09 (to appear in Mathematical Methods of Operations Research).

Alparslan Gök S.Z., Branzei R., Tijs S. (2008a) Cooperative interval games arising from airport situations with interval data. Preprint no. 107, Institute of Applied Mathematics, METU and Tilburg University, Center for Economic Research, The Netherlands, CentER DP 57.

Alparslan Gök S.Z., Branzei R., Tijs S. (2008b) Convex interval games. Preprint no. 100, Institute of Applied Mathematics, METU and Tilburg University, Center for Economic Research, The Netherlands, CentER DP 37.

Alparslan Gök S.Z., Branzei R., Tijs S. (2008c) Cores and stable sets for interval-valued games. Preprint no. 90, Institute of Applied Mathematics, METU and Tilburg University, Center for Economic Research, The Netherlands, CentER DP 17.

Alparslan Gök S.Z., Branzei R., Tijs S. (2008d) Big boss interval 
games. Preprint no. 103, Institute of Applied Mathematics, METU and Tilburg University, Center for Economic Research, The Netherlands, CentER DP 47.

Aumann R., Maschler M. (1985) Game theoretic analysis of a bankruptcy problem from the Talmud. Journal of Economic Theory 36 195-213.

Bergañtinos G., Vidal-Puga J.J. (2007) A fair rule in minimum cost spanning tree problems. Journal of Economic Theory, 137, 326-352.

Bird C.G. (1976) On cost allocation for a spanning tree: a game theoretic approach. Networks, 6, 335-350.

Branzei R., Dimitrov D., Tijs S. (2003) Shapley-like values for interval bankruptcy games. Economics Bulletin 3, 1-8.

Branzei R., Moretti S., Norde H., Tijs S. (2004) The P-value for cost sharing in minimum cost spanning tree situations. Theory and Decision, 56, 47-61.

Claus A., Kleitman D.J. (1973) Cost allocation for a spanning tree. Networks, 3, 289-304.

Curiel I., Maschler M., Tijs S. (1987) Bankruptcy games. Zeitschrift für Operations Research, 31, A143-A159.

Feltkamp V., Tijs S., Muto S. (1994) On the irreducible core and the equal remaining obligations rule of minimum cost spanning extension problems. CentER DP 1994 nr.106, Tilburg University, The Netherlands.

Montemanni R. (2006) A Benders decomposition approach for the 
robust spanning tree problem with interval data. European Journal of Operational Research, 174, 1479-1490.

Moretti S., Tijs S., Branzei R., Norde H. (2008) Cost allocation protocols for supply contract design in network situations. Mathematical Methods of Operations Research. DOI:10.1007/s00186-008-0226-9

Suijs J. (2003) Cost allocation in spanning network enterprises with stochastic connection costs. Games and Economic Behavior, 42, 156-171.

Tijs S., Branzei R., Moretti S., Norde H. (2006) Obligation rules for minimum cost spanning tree situations and their monotonicity properties, European Journal of Operational Research, 175, 121-134.

Wald A. (1950) Statistical Decision Functions. John Wiley \& Sons.

Yaman H., Karasan O.E., Pinar M. (1999) Minimum Spanning Tree Problem with Interval Data, Technical Report 9909, Department of Industrial Engineering, Bilkent University, 06533 Bilkent, Ankara, Turkey.

Yaman H., Karasan O.E., Pinar M. (2001) The robust spanning tree problem with interval data, Operations Research Letters 29, 31-40. 